\title{
Ichthyoses: Case series
}

\section{Gopal Anoop Dosemane Shrinarayana, Aneesh Samayam, Bijina Kolukulangara Dharman}

\author{
Department of Dermatology, MVJ Medical College and Research Hospital, Bangalore, India
}

Corresponding author: Dr. Gopal Anoop Dosemane Shrinarayana, E-mail: anoop1488@gmail.com

\begin{abstract}
Ichthyoses are disorders of cornification in which abnormal differentiation and desquamation of the epidermis result in a defective epidermal barrier. They can be inherited or acquired. Skin changes are clinically characterized by hyperkeratosis or scaling or both. In this series we present 2 cases of collodion baby and 3 cases of lamellar ichthyosis.
\end{abstract}

Key words: Ichthyoses; Collodion baby; Lamellar ichthyosis; Acitretin

\section{INTRODUCTION}

Ichthyoses and erythrokeratodermas are disorders of cornification in which abnormal differentiation and desquamation of the epidermis result in a defective epidermal barrier. Ichthyoses represent a large clinically and etiologically heterogeneous group of conditions that feature generalized scaling of the skin. There are congenital and acquired forms of the disease. The congenital forms include lamellar ichthyosis (LI), non-bullous congenital ichthyosiform erythroderma, and Harlequin ichthyosis; the acquired forms include ichthyosis vulgaris and X-linked ichthyosis.

\section{CASE REPORTS}

\section{Case 1}

$\mathrm{A}^{-}$term newborn infant presented after delivery due to the detection of peeling on his skin. The patient's prenatal and natal history was insignificant. The parents were $2^{\text {nd }}$ degree relatives.

\section{On Examination}

Taut, shiny collodion membrane covering the baby all over the body with erosions and fissuring at the flexures (Fig. 1). Ectropion, eclabium, sausage shaped digits and flattened ears was also seen. Other examination findings were normal.

\section{Diagnosis}

Collodion baby.

The neonate was admitted in NICU and managed with IV fluids and prophylactic antibiotics. Topical emollients were regularly applied. Fucidic acid cream was applied over the erosions. Artificial tears were applied to prevent drying of eyes.

\section{Case 2}

A term newborn infant presented after delivery due to the detection of peeling on his skin. The patient's prenatal and natal history was insignificant. There was no history of consanguinity in the family and their other child was healthy. However there is history of 2 intrauterine deaths previously.

\section{On Examination}

Taut, shiny, membrane was present covering the baby all over the body (Fig. 2). Fingers, knuckles and feet showed blanching and pallor due to taut membrane. Erosions and fissuring was seen over the flexures. Severe ectropion and eclabium was present. Other examination findings were normal.

\section{Diagnosis}

Collodion baby. 


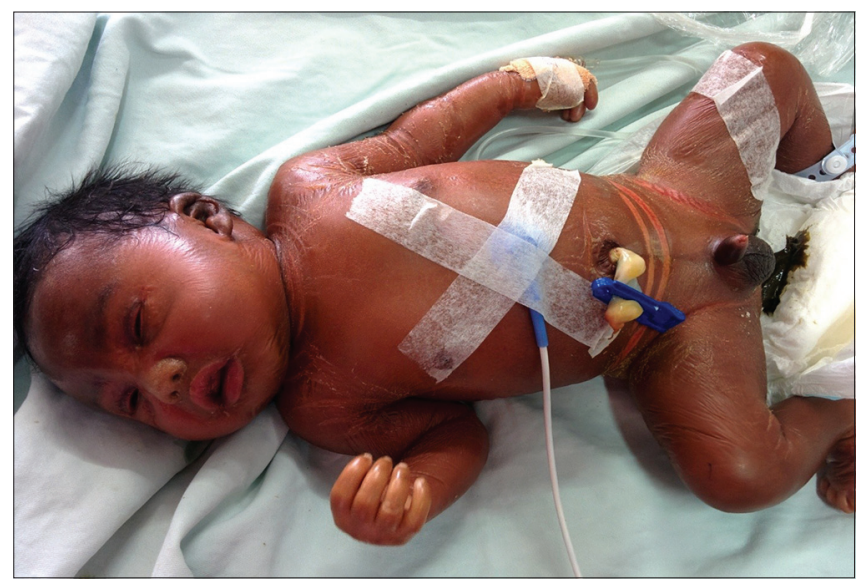

Figure 1: Taut, shiny collodion membrane covering the baby all over the body with erosions and fissuring at the flexures. Ectropion, eclabium, sausage shaped digits and flattened ears is also seen.

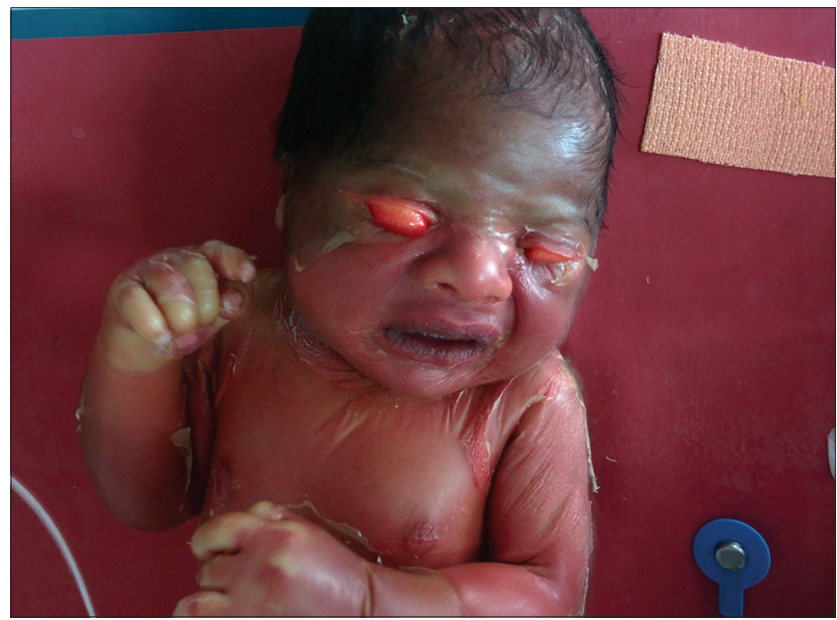

Figure 2: Taut, shiny, membrane seen covering the baby with severe ectropion and eclabium.

The neonate was admitted in NICU and managed with IV fluids and prophylactic antibiotics. Topical emollients were regularly applied. Fucidic acid cream was applied over the erosions. Artificial tears were applied to prevent drying of eyes.

\section{Case 3}

A 1 year old boy was brought to us with complaints of adherent scaling all over the body since 1 month after birth. The patient's prenatal and natal history was insignificant, with no history suggestive of collodion baby presentation at birth. Grade 4 consanguinity present in the parents. Patient's siblings are healthy.

\section{On Examination}

Multiple hyper pigmented, thick, adherent, quadrilateral scaly plaques were present all over the face, trunk, both upper limbs and lower limbs (Figs. 3a and 3b). Diffuse hyperkeratosis of the palms and soles was also noted along with generalized Xerosis. Rest of the findings were normal.

\section{Diagnosis}

Lamellar ichthyosis

The patient was treated with liberal application of emollients thrice daily. Fucidic acid cream was applied over erosions after some of the scales peeled off. Urea containing creams were applied to the scaly areas.

\section{Case 4}

A 24 year old women presented to us with complaint of dark scales all over the body since birth. History of periods of remission and aggravation of the disease was present. Her mother gives history suggestive of collodion membrane presentation in the patient at birth. There is $2^{\text {nd }}$ degree consanguinity in the parents and grandparents as well.

\section{On Examination}

Hyper pigmented, brownish-black, thick, adherent scales all present over the trunk, scalp, palms, bilateral upper limb and lower limb (Figs. 4a and 4b). Scarring alopecia was noted over the frontal and occipital region with tufts of hair left in the vertex region. Rest of the examination was normal.

\section{Diagnosis}

Lamellar ichthyosis, confirmed with biopsy.

The patient was treated with oral Acitretin $25 \mathrm{mg}$ once daily to which the patient responded very well with near complete resolution of lesions 1 month after initiation of treatment. Supportive management in the form of emollients with urea combination for application on the trunk, twice daily. Glycolic acid 6\% cream application over the face at night was also advised.

\section{Case 5}

An 8 year old girl presented to us with complaints of dark scales over both legs since 1 year. She also complained of generalized dryness of the skin and scaling over the scalp. There was history of $2^{\text {nd }}$ degree consanguinity in the parents as well. 


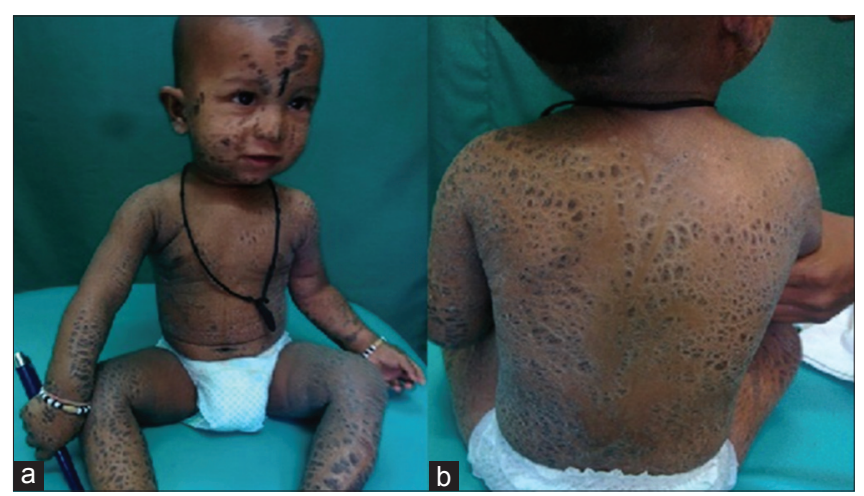

Figure 3: (a-b) Multiple hyper pigmented, thick, adherent, quadrilateral scaly plaques seen all over the face, trunk, both upper limbs and lower limbs.

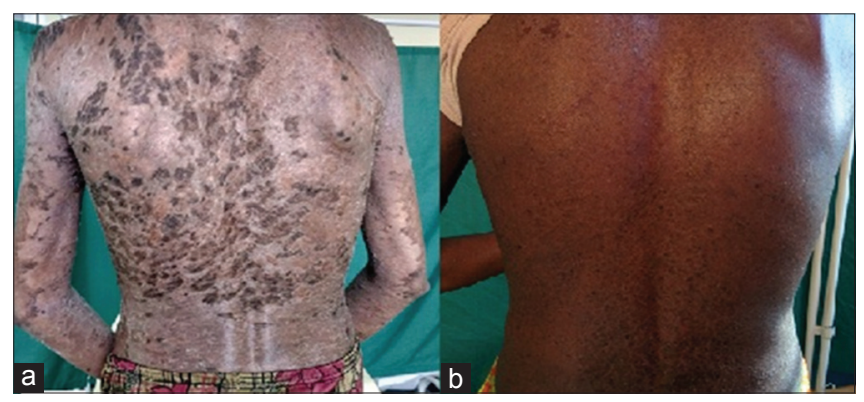

Figure 4: (a-b) Hyper pigmented, brownish-black, thick, adherent scales which cleared completely on treatment with Oral Acitretin.

\section{On Examination}

Thick, dark, brownish, adherent scales present over anterior and posterior aspect of legs bilaterally. Minimal scaling with diffuse hair loss over the scalp. Generalized xerosis and exfoliation present all over the body. Rest of the examination findings were normal.

\section{Diagnosis}

Lamellar ichthyosis, confirmed with biopsy.

The patient was treated with oral Acitretin 10mg once daily to which the patient responded very well. Liberal application of bland emollients thrice daily was also advised.

\section{DISCUSSION}

The term ichthyosis is derived from the Greek word "Ichthys' meaning "fish" and refers to the similarity in appearance of the skin to fish scales. Early reports of ichthyosis in the Indian and Chinese literature date back to several hundred years. The ichthyoses form part of a large, clinically and etiologically heterogeneous group deshmukh4of Mendelian disorders of cornification and typically involve all or most of the integument [1].

Hallopeau and Watelet were the first who gave the term Collodion baby (CB). The skin of the newborn is replaced by a cornified substance, which gives the body a parchment like appearance or a varnished appearance. This condition is inherited primarily as autosomal recessive ichthyosis either lamellar ichthyosis (LI) or Nonbullous congenital ichthyosiform erythroderma (NBCIE). $\mathrm{CB}$ is an extremely rare dermatological condition with an estimated incidence of 1 in 50,000 to 100,000 birth. A new form of the disease has been notified as "self-healing collodion syndrome' in these cases newborn completely recovers within few months after birth [2].

These infants are born with a shiny, taught, transparent collodion membrane. The membrane starts to desquamate within one to two weeks. There is usually no erythroderma, and there is no involvement of the mucosa. Due to thickened skin structure and pulling of the soft tissues around the lips and conjunctivae, ectropion and eclabium develop, which are the common diagnostic symptoms in almost all cases [3]. In our case, ectropion and eclabium were both observed.

Due to impaired skin integrity, the most significant problems for which these infants are at risk are hyperthermia, increased insensible fluid loss and electrolyte disorders, skin infections, and sepsis [4]. Sucking and pulmonary ventilation may be hindered.

It is seen that the collodion membrane sheds off in next 2-4 weeks after birth revealing the underlying skin disorder. In long term course, approximately $75 \%$ of collodion baby cases will develop an AR Congenital ichthyosis (LI or NBCIE). In just 10\% of these cases the membrane sheds off and underlying skin is normal for rest of the life termed as "Self-healing collodion baby". In rest $15 \%$ cases association with various entities is seen like ichthyosis vulgaris, trichothidystrophy, metabolic and endocrinal disorders which involve keratinization disorders gives rise to various ichthyosiform syndromes. The exact cause of the CB syndrome is not known but in most of the cases autosomal recessive inheritance pattern is seen and they are very rare and may be associated with consanguinity [5].

The first line of management is moisturizers and topical keratolytic agents, they enhances skin barrier and facilitate desquamation. Sodium chloride, urea, vitamin E acetate, glycerol and petroleum jelly are 
various agents available as moisturizers and lubricants. In severe cases with marked hyperkeratosis keratolytic agents like lactic acid, glycolic acid, salicyclic acid, $\mathrm{N}$ - acetyl- cystine, and glycol can be used. Ectropion is managed by application of artificial tears and eye lubricants. In cases with severe ectropion surgical correction is done [6]. In severe cases Acitretin $0.5-0.75 \mathrm{mg} / \mathrm{kg} /$ day can be given.

LI follows autosomal recessive transmission; its frequency is $1 / 100,000$. There is often a mutation in the gene encoding keratinocyte transglutaminase type 1 (TGM 1). TGM 1 encodes the TGAase 1 enzyme, which is one of the three TGAase enzymes found in the epidermis. This enzyme participates in the cornified envelope. In patients with TGM 1 mutation, the cornified envelope is missing, and TGAase 1 activity is reduced or nonexistent. More than 110 mutations have been reported [3]. Some of the other mutations include ABCA12, NIPAL4, ALOX12B, ALOXE3, CYP4F22, PNPLAl (OMIM 615024) [7], and CERS3 (OMIM 615023).

Clinically patients with LI present as large dark brown firmly adherent scales over the scalp, limbs and abdomen mainly, but may involve other sites also. In severely affected patients, the thick rigid scales are intermittently shed causing deep painful fissures especially around the flexures and on the digits, palms and soles. Other features include limitation of joint movements, flexion contractures, digital sclerodactyly, palmoplantar keratoderma, scarring alopecia and persistent ectropion, congenital hypoplasia of nasal and aural cartilage and impaired sweating. The child has a normal growth and intellect. Severe forms of LI seldom improve with age and psychological problems resulting from cosmetic effects and limited mobility can lead to isolation, depression and poor school performance [7].

Treatment includes emollients, keratolytics, topical calcipotriol, 10\% urea, topical $\mathrm{N}$-acetyl cysteine and topical tazarotene $0.05 \%$ gel. In severe cases Acitretin $0.5-0.75 \mathrm{mg} / \mathrm{kg} / \mathrm{day}$.

Oral retinoids have keratolytic effects that help eliminate scales and prevent excessive hyperkeratosis. Acitretin is a synthetic analog of retinoic acid. Publications regarding its successful use in ichthyosis treatment have increased in recent years. Although its mechanism of action is not precisely known, it is believed that it controls the differentiation and proliferation of the keratinized epithelium. In order to prevent relapse, treatment can take several years for some patients. Long-term use of systemic retinoids has been reported to induce teratogenic and toxic effects in the bone tissue. Bone mineralization may also be affected, and calcification frequently develops in osteophytes and ligaments. Other side effects of oral retinoids include cheilitis, dryness in the mucous membranes, mild hair loss, and itching [8].

Through starting the $0.5 \mathrm{mg} / \mathrm{kg} /$ day dose of Acitretin in our cases, the skin symptoms were significantly improved during the second week of the treatment, and by the end of the third week, the patient's skin was close to normal. The patient's tolerance for the drug was good. Side effects were not observed.

\section{CONCLUSION}

These cases are being reported for their rarity and also to highlight the association of consanguinity in them and their excellent response to oral retinoids.

\section{REFERENCES}

1. De Leonibus C, Lembo C, Santantonio A, Fioretti T, Rojo S1, Salvatore F, et al. Photoletter to the editor: Lamellar ichthyosis and arthrogryposis in a premature neonate. J Dermatol Case Rep. 2015;9:49-51.

2. Srivastava P, Srivastava A, Srivastava P, Betigeri AV, Verma M. Congenital Ichthyosis - Collodion Baby Case Report. J Clin Diagn Res. 2016;10:SJ01-2.

3. Gulasi S. Congenital Ichthyosis: A Case Treated Successfully with Acitretin. Iran J Pediatr. 2016;26:e2442.

4. Rajpopat S, Moss C, Mellerio J, Vahlquist A, Gånemo A, HellstromPigg M, et al. Harlequin ichthyosis: A review of clinical and molecular findings in 45 cases. Arch Dermatol. 2011;147:681-6.

5. Dyall-Smith D, Marks R. Dermatology at the Millennium. Informa Health Care, 1999; ISBN 1-85070-005-2: 586.

6. Harvey HB, Shaw MG, Morrell DS. Perinatal management of harlequin ichthyosis: a case report and literature review. J Perinatol. 2010;30:66-72.

7. Bubna A, Veeraraghavan M, Anandan S, Rangarajan S. A case of lamellar ichthyosis with rickets and carcinoma of the hypopharynx. Indian J Dermatol. 2014;59:634.

8. DiGiovanna JJ, Mauro T, Milstone LM, Schmuth M, Toro JR. Systemic retinoids in the management of ichthyoses and related skin types. Dermatol Ther. 2013;26:26-38.

Copyright by Gopal Anoop Dosemane Shrinarayana, et al. This is an open-access article distributed under the terms of the Creative Commons Attribution License, which permits unrestricted use, distribution, and reproduction in any medium, provided the original author and source are credited.

Source of Support: Nil, Conflict of Interest: None declared. 